\title{
POISONING BY METHYL MERCURY COMPOUNDS
}

BY

\author{
AXEL AHLMARK \\ From the Department of Industrial Hygiene, State Institute of Public Health, Stockholm, Sweden
}

(RECEIVED FOR PUBlication, MARCH 8, 1948)

In 1940 Hunter and others, reporting on cases of poisoning by methyl mercury compounds, showed that those working with certain organic mercury derivatives are exposed to great danger. In 1945 Herner collated the cases of poisoning by alkyl mercury compounds recorded in the literature. The seven cases hitherto published show a characteristic clinical picture beginning with a sensation of numbness in the tongue and weakness of the hands and feet. Motor inco-ordination followed, and the faculty of walking and the power of speech rapidly deteriorated. Defective vision and hearing occurred in several cases, and there was often mental torpidity, which led in some cases to complete dementia.

During the war of 1939-45, when Sweden was virtually cut off from the rest of the world, the manufacture of methyl mercury compounds for use in seed-dressing and wood-preserving was taken up. In consequence of this certain hygienic problems arose as well as a number of typical cases of poisoning, two of which ended fatally. A report on these experiences seems justified. First the condition of the premises will be described at the time when the accidents took place, after which a series of cases will be presented. Finally the preventive measures adopted will be discussed.

\section{The Process}

The fungicide of choice is methyl mercury hydroxide. Briefly it is produced by shaking together in ultra-violet light methyl iodide and metallic mercury, to form methyl mercury iodide. During this process the methyl iodide and the mercury are kept in glass bottles, which are opened twice every day in order to release the excess in pressure. After these have been shaken for ten or twelve days the excess of methyl iodide is evaporated off in closed vessels at from 60 to $70^{\circ} \mathrm{C}$, and the remainder of the metallic mercury is removed. The methyl mercury iodide forms a compact mass, which must be bored away before it can be emptied out of the bottles. The methyl mercury iodide is then purified by extraction with acetone in a closed system. The purified iodide is mixed with silver oxide, when the final product, methyl mercury is formed. All the above processes are carried out in a fume chamber. When opening the bottles, and on all other occasions on which methyl mercury iodide or hydroxide came into contact with air, the staff worked in masks, coats, and rubber gloves. The product has been used for seed-dressing and wood preserving in a solution of 7 per cent. or 0.25 per cent. The mixing of these final products took place in well-ventilated premises, and on these occasions too, the staff wore masks and protective clothing.

Seed-dressing was carried out in an automatically rotating drum at the place where the cases of poisoning occurred. The machine was filled and cleaned without any particular precautionary measures being taken. The seed was thrown out from the machine through a pipe on to a lower floor, where from four to six men were busy packing the seed, after which it was stored in the same room:

Wood-preserving took place at the saw-mill, by spraying a 0.25 per cent. solution of methyl mercury hydroxide on the wood. When that portion of the liquid which was not absorbed by the wood was again collected, it was strained through cloth and used again. The wood-preserving fluid was prepared in open vessels in badly ventilated premises.

\section{Case Reports}

Case 1 occurred in a factory for making methyl mercury hydroxide, Cases 2 to 4 in a seed-dressing firm, and Case 5 in a saw-mill.

Case 1.-A man of $32 \mathrm{had}$ been working for three-anda-half years in the factory where methyl mercury was manufactured. He was specially occupied with the extraction of methyl mercury iodide and also with releasing the excess pressure in the bottles in which the methyl mercury iodide had formed. He was extremely careful in his work and observed all the protective precautions in detail. At the beginning of April, 1944, he began to complain of giddiness, his fingers felt " funny," and the tips became numb. In the middle of 
May he began to speak indistinctly and complained of numbness in the tongue. He began to walk unsteadily and found it difficult to keep his balance. Towards the end of May, owing to his trembling hands, he could not even drink a cup of coffee without help. He became irritable, and lost much weight though there were no intestinal symptoms. He was at first regarded as being hysterical. He was admitted to hospital on June 1, 1944, after which his condition deteriorated rapidly, and by the end of the month he could not stand upright. The ataxia increased. He felt a numbness in the hands, the tongue, and round the mouth, though it was not possible definitely to prove any defect in sensibility. His hearing became worse, and his speech was barely understandable. He could scarcely write at all. His face took on an almost euphoric expression. From the beginning of July his condition improved, the ataxia decreased, and the hearing improved. By October, 1944, he was able to feed himself. In January, 1948, he was still suffering from ataxia and could not eat properly. He had not recovered mentally. (For more details see Herner, 1945.)

\section{TABLE}

MERCURY CONTENT $(\mu \mathrm{g} / \mathrm{G})$ OF ORGANS TAKEN FROM FATAL CASES OF METHYL MERCURY POISONING

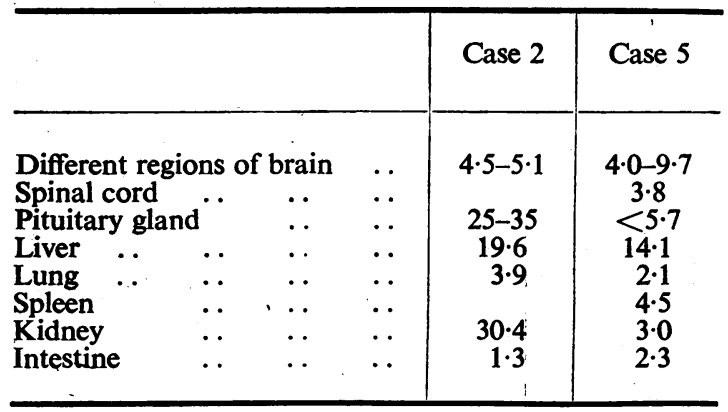

Case 2.-A man of 23, from Aug. 16 to Sept. 16, 1944, had been occupied in the packing of a seed-dressing containing methyl mercury. He was taken ill about a month after the work was completed and felt a numbness and a stinging sensation in the hands. Towards the end of October in the same year it was difficult for him to keep his balance and to walk, and he noticed that he could not hold a filled glass steadily. He began to speak indistinctly. On many occasions he suffered from diplopia. According to his family his temper had deteriorated before he was admitted to hospital on Oct. 31, 1944. He was then found to have an unsteady walk, and he moved clumsily and insecurely. From Nov. 4 onwards the patient gradually got worse, throwing himself about uneasily in bed and holding his arms and legs in strangely stereotyped positions. On Nov. 8 there was extreme motor unrest which increased, and he died the following day. At first the patient was thought to have encephalitis. Analysis of some of the organs showed high figures for mercury (see Table).

Case 3.-A man of 22 had the same work as Case 2. On about Nov. 4, 1944, he began to feel a tingling sensation in his hands, feet, and round his mouth, and this continued for about a couple of weeks. There were no other symptoms.

Case 4.-A man of 18 had the same work as Cases 2 and 3. On Nov. 5, 1944, the patient noticed a tingling sensation and a numbness in the fingers; which improved quickly at first, but he still felt it to a certain extent on Dec. 5, 1944. There were no other symptoms.

Case 5.-A man of 57, for five years had on repeated occasions impregnated wood with a methyl mercury preparation. He was taken ill in the middle of December, 1944 , with a numbness in the hands and forearms, digital inco-ordination, dizziness, and unsteady walk. He found it difficult to hold his coffee cup steadily, and on one occasion he fell off his bicycle for no apparent reason. The symptoms gradually increased and the patient was admitted to hospital on Dec. 16, 1944.

It was soon impossible to understand what he said. He developed concentric scotomata of the fields of vision and later became completely blind. Towards the end of the month he got more or less uneasy, alternating with periods of deep apathy. He had to be fed through a tube, but he could swallow water. He died on Jan. 2 after a terminal rise of temperature. Before the similarity of Cases 1 to 4 had been fully realized, the condition was interpreted as one of cerebral tumour and the patient was transferred to the neurosurgical department. Analysis of the organs showed high figures for mercury (see Table).

The symptoms here referred to show a striking resemblance to those of cases of poisoning by alkyl mercury compounds previously described. Thanks to these, and especially through the investigations made in two of the cases of the concentration of mercury found in various organs, it has been possible definitely to establish the ætiology.

\section{Preventive Measures}

Because of the occurrence of these cases and others, the protective measures at the places of work were improved in many respects.

At places where seed-dressing was being carried out, steps were taken as far as possible to prevent the workmen from inhaling dust and vapours, and to prevent the chemical compounds from coming into contact with the skin. On our advice these steps have been enforced by the state authorities responsible for laws and regulations concerning the safety of the workers. Thus, the seed-dressing plant must be built in particularly well-ventilated rooms, with special suction arrangements to prevent the dissemination of dust and vapours about the premises when the machines are being filled with products containing mercury. The spread of dust must be carefully avoided, and it is advisable to pour in liquids through tubes. When apparatus is cleaned, respirators must be used and both gloves and aprons must be worn to prevent absorption. 
Places where the seed is packed must likewise be specially enclosed and well ventilated.

Steps must also be taken to afford workmen employed on mercury impregnation every possibility of satisfactory personal hygiene. A sufficient number of lavatories must always be available. Special clothes and foot-gear must also be worn during work. Meals must not be served at the place where work is being carried on, and when meals are taken clothes must be changed and careful attention paid to personal hygiene. Corresponding arrangements must also be made at places where methyl mercury is used for preserving wood.

Taking into consideration the high degree of toxicity of these compounds, these technical protective measures are not sufficient. It is important to remember that the smallest changes in the arrangements of the plant or in the order of the work may involve risk. It is certainly necessary to analyse the mercury concentration in the air repeatedly and to have the staff regularly examined by a physician.

No conclusion has been arrived at as to what is the upper limit of safe exposure to these mercury compounds in the air. Considering the extremely high toxic effect of metallic mercury, and bearing in mind that some authors give 0.06 to $0.20 \mathrm{mg}$. per cubic metre as an allowable concentration, a percentage higher than $0.01 \mathrm{mg}$. per cubic metre of methyl mercury compounds should not be allowed.

In order to test the effectivity of the protective steps taken at the factory for the manufacture of methyl mercury compounds a regular medical control of the staff is carried out. This consists of a monthly physical and neurological examination in which careful enquiry is made of early symptoms. Such symptoms scarcely differ from those generally found in neurasthenics when they think that they have been exposed to toxic risks. It has therefore proved extremely difficult in the case of sensitive persons to distinguish between true mercury effects and neurological symptoms having quite a different origin. In order to overcome these difficulties attempts have been made to prevent persons inclined to neurasthenia from carrying out dangerous work. Consequently, before taking up duties in the factory everyone must undergo a thorough medical examination, after which only absolutely healthy persons without any predisposition to neurasthenia are allowed to work on the premises where mercury compounds are handled. By estimating the mercury content of the blood and urine, it has been possible to check the position to a certain degree. In a considerable number of cases the urine rates have been between 30 and $60 \mu \mathrm{g}$ mercury/litre urine without the person in question showing the least sign of being affected. On the other hand, there have been some cases with unmistakable but slight signs of mercury effect with a percentage of 50 to $100 \mu \mathrm{g}$ mercury/litre urine.

From these investigations the conclusion has been drawn that a person should not be allowed to undertake work with methyl mercury compounds which produces a mercury excretion in the urine exceeding 10 to $15 \mu \mathrm{g}$ litre. Fortunately it has been found possible to attain this.

\section{Summary}

A description is given of five cases of methyl mercury poisoning observed at a factory for the manufacture of such compounds, in a seed-dressing plant and at a saw-mill. The cases show a striking resemblance to seven others previously described in the literature. An account is given of the steps, both technical and medical, that have been taken to protect the staff from the risks of poisoning. Particular attention must be paid to repeated environmental and medical examinations, including both blood and urine tests for mercury frequently repeated during the period of exposure.

I wish to express my gratitude to $R$. Vesterberg for his kind help with the analyses.

\section{REFERENCES}

Herner, T. (1945). Nord. Med., 26, 833

Hunter, D., Bomford, R. R., and Russell, D. S. (1940). Quart. J. Med., n.s., 9, 193. 